\title{
DISPERSIVE ORDERING AND MONOTONE FAILURE RATE DISTRIBUTIONS
}

\author{
J. BARTOSZEWICZ, ${ }^{*}$ University of Wrockaw
}

\begin{abstract}
Recently many authors (e.g. Shaked (1982), Deshpande and Kochar (1983), Sathe (1984)) have established relations between the dispersive ordering and some other partial orderings of distributions. This note presents connections which the dispersive ordering has with monotone failure rate distributions.
\end{abstract}

PARTIAL ORDERING

Recently many authors have studied properties of the dispersive ordering of distributions defined by Lewis and Thompson (1981) and Shaked (1982). Let $F$ and $G$ be two cumulative distribution functions with $F^{-1}$ and $G^{-1}$ as the respective left-continuous inverses. Then $F$ is said to be dispersed with respect to $G(F \stackrel{\text { disp }}{<} G)$ if and only if

$$
F^{-1}(\beta)-F^{-1}(\alpha) \leqq G^{-1}(\beta)-G^{-1}(\alpha) \text { whenever } 0<\alpha<\beta<1 \text {. }
$$

Shaked (1982) has proved that $F \stackrel{\text { disp }}{<} G$ if and only if

$$
G^{-1} F(x)-x \text { is non-decreasing in } x .
$$

He has also remarked that for $F$ and $G$ absolutely continuous with corresponding densities $f$ and $g, F \stackrel{\text { disp }}{<} G$ if and only if $g\left(G^{-1}(u)\right) \leqq f\left(F^{-1}(u)\right)$ whenever $u \in(0,1)$. Deshpande and Kochar (1983) have noticed that the condition (1) had already been used by Doksum (1969) for defining so-called tail-ordering. Thus dispersive ordering is the same as tail-ordering.

Many authors have established connections between this ordering and other partial orderings of distributions. Shaked (1982) has proved that if distributions $F$ and $G$ have the support $[0, \infty)$ and $F(0)=G(0)=0$, then $F \stackrel{\text { disp }}{<} G$ implies $F \stackrel{\text { st }}{\leqq} G$, i.e. $F(x) \geqq G(x)$ for all $x$. In reliability theory there are two well-known orderings of distributions (see for example Barlow and Proschan (1975)). Let $F$ and $G$ be continuous and strictly increasing on their supports $S_{F}$ and $S_{G}$ being intervals and $F(0)=G(0)=0 . F$ is said to be convex-ordered with respect to $G(F \mathcal{<} G)$ if and only if $G^{-1} F$ is a convex function on $S_{\mathrm{F}} . F$ is said to be star-ordered with respect to $G(F \stackrel{*}{*} G)$ if and only if $G^{-1} F$ is a star-shaped function on $S_{\mathrm{F}}$, i.e. $G^{-1} F(x) / x$ is non-decreasing on $S_{\mathrm{F}}$. It is easily seen that

Received 4 December 1984.

Postal address: Mathematical Institute, University of Wrocław, Pl. Grunwaldzki 2/4, 50-384 Wrocław, Poland. 
$F \stackrel{c}{<} G$ implies $F$ 光 $G$. Doksum (1969) and Deshpande and Kochar (1983) have proved that if $F$ and $G$ are absolutely continuous, $F(0)=G(0)=0$ and the corresponding densities are such that $f(0) \geqq g(0)>0$, then $F \stackrel{*}{<} G$ implies $F \stackrel{\text { disp }}{<} G$. Sathe (1984) has pointed out that $\lim _{x \rightarrow 0^{+}}\left(G^{-1} F(x) / x\right) \geqq 1$ is the only condition required to imply that $F \stackrel{\text { disp }}{<} G$ if $F \stackrel{*}{<} G$. From Sathe's proof it directly follows that if $F \stackrel{\text { st }}{\leqq} G$ and $F \stackrel{*}{<} G$, then $\stackrel{\text { disp }}{<} G$. Bartoszewicz (1982) has proved that if $S_{F}=\left[0, a_{1}\right], S_{G}=\left[0, a_{2}\right], 0<a_{1} \leqq a_{2} \leqq \infty$ and $F \stackrel{c}{<} G$ or $S_{F}=[0, \infty), S_{G}=[b, \infty), G(b)=0, b \geqq 0$, and $G \stackrel{c}{<} F$, then $F \stackrel{\text { st }}{=} G$ implies $F \stackrel{\text { disp }}{<}$.

In this note we present some connections between dispersive ordering and increasing failure rate (IFR) and decreasing failure rate (DFR) distributions. Assume now that $F$ and $G$ are continuous and strictly increasing on their support $[0, \infty)$ and $F(0)=G(0)=0$. Denote by

$$
\bar{F}(x \mid t)=\frac{1-F(t+x)}{1-F(t)}, \quad x \geqq 0, \quad t \geqq 0,
$$

the conditional reliability of a unit of age $t$ if $F$ is the life distribution of the unit. $\bar{G}(x \mid t)$ is analogously defined. $F$ is said to be an IFR (DFR) distribution if $\bar{F}(x \mid t)$ is non-increasing (non-decreasing) in $t \geqq 0$ for each $x \geqq 0$ (see e.g. Barlow and Proschan (1975)).

Under the above assumptions the following theorems hold.

Theorem 1. If $\bar{F}(x \mid t) \leqq \bar{G}(x \mid t)$ for every $x \geqq 0$ and each $t \geqq 0$ and $F$ or $G$ is DFR, then $F \stackrel{\text { disp }}{<} G$.

Proof. Assume $G$ is DFR. $\bar{F}(x \mid t) \leqq \bar{G}(x \mid t)$ for $x \geqq 0$ and $t \geqq 0$ implies $F(x) \geqq G(x)$ for $x \geqq 0$. Hence

$$
F^{-1} G(x) \leqq x \leqq G^{-1} F(x), \quad x \geqq 0 .
$$

Since $G$ is DFR, (2) implies

$$
\begin{aligned}
& \bar{F}(x \mid t) \leqq \bar{G}(x \mid t) \leqq \bar{G}\left(x \mid G^{-1} F(t)\right), \quad x \geqq 0, t \geqq 0 \\
& \quad \Rightarrow F(x+t) \geqq G\left(G^{-1} F(t)+x\right), \quad x \geqq 0, t \geqq 0 \\
& \quad \Leftrightarrow G^{-1} F(x+t)-(x+t) \geqq G^{-1} F(t)-t, \quad x \geqq 0, t \geqq 0 \\
& \Leftrightarrow G^{-1} F(x)-x \text { non-decreasing in } x \geqq 0 \Leftrightarrow F \stackrel{\text { disp }}{<} .
\end{aligned}
$$

The proof runs similarly if $F$ is assumed to be DFR.

Theorem 2. If $F \stackrel{\text { disp }}{<} G$ and $F$ or $G$ is IFR, then $\bar{F}(x \mid t) \leqq \bar{G}(x \mid t)$ for every $x \geqq 0$ and each $t \geqq 0$.

Proof. Assume $G$ is IFR.

$$
\begin{aligned}
F \stackrel{\text { disp }}{<} G & \Leftrightarrow G^{-1} F(x)-x \text { non-decreasing in } x \\
& \Leftrightarrow G^{-1} F(x+t) \geqq G^{-1} F(t)+x, \quad x \geqq 0, t \geqq 0 \\
& \Leftrightarrow F(x+t) \geqq G\left(G^{-1} F(t)+x\right) \quad x \geqq 0, t \geqq 0 .
\end{aligned}
$$


Since $F \stackrel{\text { disp }}{<} G$ implies (2) and $G$ is IFR, then from (3) it follows

$$
\frac{1-G(t+x)}{1-G(t)} \geqq \frac{1-G\left(G^{-1} F(t)+x\right)}{1-G\left(G^{-1} F(t)\right)} \geqq \frac{1-F(t+x)}{1-F(t)}, \quad x \geqq 0, \quad t \geqq 0,
$$

i.e. $\bar{F}(x \mid t) \leqq \bar{G}(x \mid t), x \geqq 0, t \geqq 0$.

The proof is similar when $F$ is assumed to be IFR.

If the distributions $F$ and $G$ have corresponding densities $f$ and $g$, one defines the failure rate functions

$$
r_{F}(t)=\frac{f(t)}{1-F(t)} \quad \text { and } \quad r_{G}(t)=\frac{g(t)}{1-G(t)}, \quad t \geqq 0 .
$$

Then one can easily prove the following versions of Theorems 1 and 2 .

Theorem $1^{\prime}$. Let $F$ and $G$ be absolutely continuous on their support $[0, \infty)$ and $F(0)=G(0)=0$. If $r_{G}(t) \leqq r_{F}(t)$ for every $t \geqq 0$ and $F$ or $G$ is DFR, then $F<G$.

Theorem 2 '. Let $F$ and $G$ be absolutely continuous on their support $[0, \infty)$ and $F(0)=G(0)=0$. If $F \stackrel{\text { disp }}{<} G$ and $F$ or $G$ is IFR, then $r_{G}(t) \leqq r_{F}(t)$ for every $t \geqq 0$.

\section{References}

Barlow, R. E. AND Proschan, F. (1975) Statistical Theory of Reliability and Life Testing. Holt, Rinehart and Winston, New York.

BARTOSZEWICZ, J. (1982) Moment inequalities for order statistics from ordered families of distributions. Metrika. To appear.

DESHPANDE, J. V. AND KOCHAR, S. C. (1983) Dispersive ordering is the same as tail-ordering. Adv. Appl. Prob. 15, 686-687.

DoksuM, K. (1969) Star-shaped transformations and the power of rank tests. Ann. Math. Statist. 40, 1167-1176.

LeWIS, T. AND ThOMPson, J. W. (1981) Dispersive distributions and the connection between dispersivity and strong unimodality. J. Appl. Prob. 18, 76-90.

Sathe, Y. (1984) A comment on star-ordering and tail-ordering. Adv. Appl. Prob. 16, 692.

SHAKeD, M. (1982) Dispersive ordering of distributions. J. Appl. Prob. 19, 310-320. 